\title{
Subdifferentiability of the norm and the Banach-Stone Theorem for real and complex JB*-triples
}

\author{
Julio Becerra Guerrero and Antonio M. Peralta*
}

Departamento Matemática Aplicada, Facultad de Ciencias, Universidad de Granada, 18071 Granada, Spain.

E-mail: juliobg@ugr.es

Departamento de Análisis Matemático, Facultad de Ciencias, Universidad de Granada, 18071 Granada, Spain.

E-mail: aperalta@ugr.es

2000 Mathematics Subject Classification: 46B04, 46L05 and 46L70.

\begin{abstract}
We study the points of strong subdifferentiability for the norm of a real $\mathrm{JB}^{*}$-triple. As a consequence we describe weakly compact real $\mathrm{JB}^{*}$-triples and rediscover the Banach-Stone Theorem for complex $\mathrm{JB}^{*}$-triples.
\end{abstract}

\section{Introduction}

Let $X$ be a Banach space. The norm of $X$ is said to be strongly-subdifferentiable at a morn-one point $x \in X$ whenever the limit

$$
\lim _{\alpha \rightarrow 0^{+}} \frac{\|x+\alpha y\|-1}{\alpha}
$$

exists uniformly for $y$ in the closed unit ball of $X$. The points of strong subdifferentiability for the norm of a $\mathrm{C}^{*}$-algebra were characterized by Contreras, Payá and Werner in [6]. Recently, Becerra-Guerrero and Rodríguez-Palacios

*Authors Partially supported by I+D MCYT projects no. BFM2002-01529 and BFM2002-01810, and Junta de Andalucía grant FQM 0199 
have completely described the points of strong subdifferentiability for the norm of a (complex) $\mathrm{JB}^{*}$-triple [3]. In the latter work, the authors show that the norm of a $\mathrm{JB}^{*}$-triple $\mathcal{E}$ is strongly subdifferentiable at a norm-one point $x$ if and only if 1 is an isolated point of the triple spectrum of $x$, if and only if the support tripotent of $x$ in the bidual, $\mathcal{E}^{* *}$, of $\mathcal{E}$ lies in $\mathcal{E}$. As a consequence, the authors show that the JB*-triples whose norms are strongly subdifferentiable at every point of their unit spheres are precisely the so-called weakly compact $\mathrm{JB}^{*}$-triples.

The aim of the present paper is to describe the points of strong subdifferentiability of the norm of a real JB*-triple (see definition bellow). In our main result (Theorem 2.4) we prove that the norm of a real $\mathrm{JB}^{*}$-triple $E$ is strongly subdifferentiable at a norm-one point $x$ if and only if the (unique) norm becoming its complexification a complex $\mathrm{JB}^{*}$-triple is strongly subdifferentiable at $x$. As a consequence we characterize, in Corollary 2.5, the points of strong subdifferentiability for the norm of a real JB*-triple, extending the description provided by Becerra-Guerrero and Rodríguez-Palacios in the complex setting.

In [23] Werner showed that the characterization of the points of strong subdifferentiability for the norm of $\mathrm{C}^{*}$-algebra can be applied to obtain an alternative proof of the non-commutative Banach-Stone Theorem provided by Kadison: "The linear surjective isometries from a unital $\mathrm{C}^{*}$-algebras $A$ onto another unital $\mathrm{C}^{*}$-algebra $B$ are precisely of the form $x \mapsto u \Phi(x)$, where $u$ is a unitary element of $B$ and $\Phi$ is a Jordan isomorphism from $A$ onto $B$. In the already quoted paper (see [23, Remarks 3.]) the author establishes without proof that it is possible to extend the method he applied to the more general setting of $\mathrm{JB}^{*}$-triples. In the last section of this paper we include a complete extension of Werner's method to the setting of real and complex $\mathrm{JB}^{*}$-triples and the appropriated version of the Banach-Stone theorem for $\mathrm{JB}^{*}$-triples.

\section{Main result}

Given a Banach space $X$, we denote by $B_{X}, S_{X}$, and $X^{*}$ the closed unit ball, the unit sphere, and the dual space of $X$, respectively.

Let $x$ be a norm one element in a Banach space $X$. The set $D(X, x)$ of all states of $X$ relative to $x$ is define by

$$
D(X, x):=\left\{f \in S_{X^{*}}: f(x)=\|x\|\right\} .
$$


A $J B^{*}$-triple is a complex Banach space $\mathcal{E}$ equipped with a continuous triple product

$$
\begin{gathered}
\{., ., .\}: \mathcal{E} \otimes \mathcal{E} \otimes \mathcal{E} \rightarrow \mathcal{E} \\
(x, y, z) \mapsto\{x, y, z\}
\end{gathered}
$$

which is bilinear and symmetric in the outer variables and conjugate linear in the middle one and satisfies:

(a) (Jordan Identity)

$$
L(x, y)\{a, b, c\}=\{L(x, y) a, b, c\}-\{a, L(y, x) b, c\}+\{a, b, L(x, y) c\},
$$

for all $x, y, a, b, c \in \mathcal{E}$, where $L(x, y): \mathcal{E} \rightarrow \mathcal{E}$ is the linear mapping given by $L(x, y) z=\{x, y, z\}$;

(b) The map $L(x, x)$ is an hermitian operator with non-negative spectrum for all $x \in \mathcal{E}$;

(c) $\|\{x, x, x\}\|=\|x\|^{3}$ for all $x \in \mathcal{E}$.

Every $\mathrm{C}^{*}$-algebra is a $\mathrm{JB}^{*}$-triple with respect to $\{x, y, z\}=2^{-1}\left(x y^{*} z+\right.$ $\left.z y^{*} x\right)$, every $\mathrm{JB}^{*}$-algebra is a $\mathrm{JB}^{*}$-triple with triple product $\{a, b, c\}=(a \circ$ $\left.b^{*}\right) \circ c+\left(c \circ b^{*}\right) \circ a-(a \circ c) \circ b^{*}$, and the Banach space $B(H, K)$ of all bounded linear operators between two complex Hilbert spaces $H, K$ is also an example of a $\mathrm{JB}^{*}$-triple with respect to $\{R, S, T\}=2^{-1}\left(R S^{*} T+T S^{*} R\right)$.

Let $X$ be a complex Banach space with a conjugation (conjugate linear isometry of period two) $\tau$ on $X$. We will denote by $X^{\tau}$ the real Banach subspace of $X$ of all $\tau$-fixed points in $X$. In this case we will say that $X^{\tau}$ is a real form of $X$.

It is worth mentioning that a real $\mathrm{JB}^{*}$-triple is a norm-closed real subtriple of a $\mathrm{JB}^{*}$-triple [16, Definition 2.1]. Let $E$ be a real $\mathrm{JB}^{*}$-triple. By [16, Proposition 2.8], there exists a unique complex $\mathrm{JB}^{*}$-triple structure on the algebraic complexification $E \oplus i E$ (denoted by $\widehat{E}$ ) and a conjugation $\tau$ on $E+i E$ such that $E=\widehat{E}^{\tau}:=\{z \in \widehat{E}: \tau(z)=z\}$, i.e., every real JB*-triple is a real form of its complexification, which is a complex JB*-triple. Every real $\mathrm{C}^{*}$-algebra, every real Hilbert space, every complex $\mathrm{JB}^{*}$-triple (when is regarded as a real Banach space) and the Banach space of all bounded linear operators between real Hilbert spaces are examples of real $\mathrm{JB}^{*}$-triples (cf. [16]).

By a real or complex $\mathrm{JBW}^{*}$-triple we mean a real or complex $\mathrm{JB}^{*}$-triple which is also a dual Banach space whose triple product is separately weak*continuous [16, §4]. By [20] and [1] we know that the assumption of the separate weak ${ }^{*}$-continuity is redundant. The bidual $E^{* *}$ of every real or complex 
$\mathrm{JB}^{*}$-triple is a $\mathrm{JBW}^{*}$-triple with triple product extending the product of $E$ (cf. [8] and [16, Lemma 4.2], respectively).

Let $U$ be a real or complex $\mathrm{JB}^{*}$-triple and let $e$ be a tripotent in $U$ (i.e. $\{e, e, e\}=e)$. It is known that $U$ admits the following decomposition in terms of the eigenspaces of $L(e, e)$,

$$
U=U_{0}(e) \oplus U_{1}(e) \oplus U_{2}(e),
$$

where $U_{k}(e):=\left\{x \in U: L(e, e) x=\frac{k}{2} x\right\}$ is a subtriple of $U(k: 0,1,2)$. The natural projection of $U$ onto $U_{k}(e)$ will be denoted by $P_{k}(e)$. This decomposition is the so-called Peirce decomposition with respect to the tripotent $e$ and the natural projections are the so-called Peirce projections. The following rules, known as Peirce rules, are also satisfied

$$
\begin{gathered}
\left\{U_{k}(e), U_{l}(e), U_{m}(e)\right\} \subseteq U_{k-l+m}(e), \\
\left\{U_{0}(e), U_{2}(e), U\right\}=\left\{U_{2}(e), U_{0}(e), U\right\}=0,
\end{gathered}
$$

where $U_{k-l+m}(e)=0$ whenever $k-l+m \neq 0,1,2$.

A tripotent $e$ in a real or complex $\mathrm{JB}^{*}$-triple $U$ is called minimal whenever $U^{1}(e)=\mathbb{R} e$, where $U^{1}(e)=\{x \in U: Q(e)(x)=x\}$.

Let $E$ be a real $\mathrm{JB}^{*}$-triple with complexification $\widehat{E}$ and let $e$ be a tripotent in $E$. It is clear that the Peirce projections of $E$ with respect to $e$ coincide with the restrictions to $E$ of the Peirce projections of $\widehat{E}$ with respect to $e$. Therefore, the following result follows from [14, Lemma 1.3 and Lemma 1.6].

Lemma 2.1. Let e be a tripotent in a real $J B^{*}$-triple E. Then we have

(a) $\left\|P_{2}(e)(x)+P_{0}(e)(x)\right\|=\max \left\{\left\|P_{2}(e)(x)\right\|,\left\|P_{0}(e)(x)\right\|\right\}$, for all $x \in E$;

(b) $\left\|P_{2}(e)^{*}(f)+P_{0}(e)^{*}(f)\right\|=\left\|P_{2}(e)^{*}(f)\right\|+\left\|P_{0}(e)^{*}(f)\right\|$, for all $f \in E^{*}$.

(c) If $x$ is a norm-one element in $E$ with $P_{2}(e)(x)=e$, then $P_{1}(e)(x)=0$, thus $x=e+P_{0}(e)(x)$.

Let $X$ be a complex Banach space with a conjugation $\tau$ on $X$. Then $\tau$ can be extended to a conjugation $\tilde{\tau}$ on $X^{*}$ in the following way

$$
\begin{gathered}
\tilde{\tau}: X^{*} \rightarrow X^{*} \\
\tilde{\tau}(f)(x)=\overline{f(\tau(x))}\left(f \in X^{*}\right) .
\end{gathered}
$$


In this case, it is also known that $\left(X^{*}\right)^{\tilde{\tau}}$ is isometric to $\left(X^{\tau}\right)^{*}$ via $f \mapsto$ $\left.f\right|_{X^{\tau}}$. Under this identification, $X^{*}$ admits the decomposition $X^{*}=\left(X^{\tau}\right)^{*}+$ $i\left(X^{\tau}\right)^{*}$. Let $x \in S_{X^{\tau}} \subset S_{X}$ and $f \in D(X, x)$. Then

$$
\tilde{\tau}(f)(x)=\overline{f(\tau(x))}=\overline{f(x)}=1,
$$

which shows that $\tilde{\tau}(f) \in D(X, x)$. Therefore $\tilde{\tau}(D(X, x))=D(X, x)$ and hence $D\left(X^{\tau}, x\right)=D(X, x)^{\tilde{\tau}}$.

Having in mind that every tripotent in a real $\mathrm{JB}^{*}$-triple is clearly a tripotent in its complexification and the strong subdifferentiability at a norm-one point is inherited by real subspaces, the next corollary is a consequence of Theorem [3, Theorem 2.7].

Corollary 2.2. Let $E$ be a real $J B^{*}$-triple. Then the norm of $E$ is strongly subdifferentiable at every tripotent in $E$.

Let $x$ be a norm-one element in a real or complex $\mathrm{JBW}^{*}$-triple $U$. The set $D(U, x) \cap U_{*}$ is a (possibly empty) proper closed face of $B_{U_{*}}$, and therefore, by [11, Theorem 3.7 and Lemma 2.1], there exists a unique tripotent $u$ (possibly equal to zero) in $U$ so that $D(U, x) \cap U_{*}=D(U, u) \cap U_{*}$. Such a tripotent is called the support tripotent of $x$ and will be denoted by $u(U, x)$. Let $E$ be a real $\mathrm{JBW}^{*}$-triple and let $x \in S_{E}$. As we have seen above, $E=\widehat{E}^{\tau}$ where $\widehat{E}$ is the complexification of $E$ and $\tau$ is a conjugation on $\widehat{E}$. By [9, Lemma 3.4], $u(\widehat{E}, x)$ is the limit in the weak*-topology of the sequence $\left(x^{2 n+1}\right)$, where $x^{2 n+1}$ is inductively defined by $x^{3}=\{x, x, x\}$ and $x^{2 n+1}=\left\{x, x^{2 n-1}, x\right\}$. Since the canonical conjugation on $\widehat{E}$ is weak*-continuous and preserves the triple product we have $\tau(u(\widehat{E}, x))=u(\widehat{E}, x)$. Now, [11, Theorem 3.7] ascertains that $u(E, x)=u(\widehat{E}, x)$.

Having the above facts in mind, the same arguments given in $[3$, Theorem $2.5]$ can be adapted to obtain the following corollary.

Corollary 2.3. Let $E$ be a real $J B W^{*}$-triple, and let $x$ be in $S_{E}$. The norm is strongly subdifferentiable at $x$ if and only if $D(E, x) \cap E_{*}$ is weak*-dense in $D(E, x)$.

We can now establish our main result.

Theorem 2.4. Let $E$ be a real $J B^{*}$-triple with complexification $\widehat{E}$ and let $x$ be in $S_{E}$. Then the norm of $E$ is strongly subdifferentiable at $x$ if and only if the same conclusion holds for the norm of $\widehat{E}$. 
Proof. We have already mentioned that the strong subdifferentiability of the norm at a norm-one point is inherited by real subspaces. In order to see the other implication let us suppose that the norm of $E$ is subdifferentiable at $x \in S_{E}$.

Let $u=u\left(E^{* *}, x\right)$ be the support tripotent of $x$ in $E^{* *}$. Then

$$
D(E, x)=D\left(E^{* *}, u\right) \cap E^{*} .
$$

Since the norm of $E$ is strongly subdifferentiable at $x$ we deduce that the same conclusion remains true for the norm of $E^{* *}$ [15, Corollary 2.1], and hence, by Corollary 2.3, we get

$$
\overline{D(E, x)} w^{*}=D\left(E^{* *}, x\right) .
$$

By Corollary 2.2 and Corollary 2.3 we also have

$$
\overline{D\left(E^{* *}, u\right) \cap E^{*}} w^{*}=D\left(E^{* *}, u\right) .
$$

It follows by $(1)$ and $(2)$ that $D\left(E^{* *}, x\right)=D\left(E^{* *}, u\right)$. By [11, Theorem 3.9] it follows that $x \in u+B_{E_{0}^{* *}(u)}$. We claim that $\left\|P_{0}(u)(x)\right\|<1$. Otherwise, by Lemma 2.1 and the Hahn-Banach theorem, there exist an element in $D\left(E^{* *}, x\right) \backslash D\left(E^{* *}, u\right)$, which is impossible. Therefore $x=u+P_{0}(u)(x)$ with $\left\|P_{0}(u)(x)\right\|<1$. Finally, by Peirce rules, $x^{2 n+1}=u+\left(P_{0}(u)(x)\right)^{2 n+1}$, thus,

$$
\left\|x^{2 n+1}-u\right\| \leq\left\|P_{0}(u)(x)\right\|^{2 n+1} \rightarrow 0 .
$$

Therefore $u \in E$. Since $u=u\left(E^{* *}, x\right)=u\left((\widehat{E})^{* *}, x\right) \in E \subseteq \widehat{E}$, Theorem [3, Theorem 2.7, (4) $\Rightarrow(1)$ ] implies that the norm of $\widehat{E}$ is strongly subdifferentiable at $x$.

Let $x$ be an element in a complex $\mathrm{JB}^{*}$-triple $\mathcal{E}$, and denote by $\mathcal{E}(x)$ the $\mathrm{JB}^{*}$-subtriple of $\mathcal{E}$ generated by $x$. It is known that there exists a locally compact subset $S_{x}$ of $(0,+\infty)$ such that $S_{x} \cup\{0\}$ is compact and $\mathcal{E}(x)$ is $\mathrm{JB}^{*}$-triple isomorphic to the $\mathrm{C}^{*}$-algebra $C_{0}\left(S_{x}\right)$ under a triple isomorphism $\Psi$, which satisfies $\Psi(x)(t)=t\left(t \in S_{x}\right)$ (cf. [17, 4.8], [18, 1.15] and [14]). The subset $S_{x}$ is called the triple spectrum of $x$. When $x$ is an element in a real $\mathrm{JB}^{*}$-triple $E$, the complex triple spectrum of $x$ is the triple spectrum of $x$ when $x$ is regarded as an element in the complexification, $\widehat{E}$, of $E$.

In the case of a real $\mathrm{JB}^{*}$-triple we can obtain the following characterizations of the strong subdifferentiability of its norm at a point of the unit sphere, similar to those obtained for (complex) $\mathrm{JB}^{*}$-triples in $[3$, Theorem $2.7]$. 
Corollary 2.5. Let $E$ be a real $J B^{*}$-triple. The following assertions are equivalent for an element $x$ in the unit sphere of $E$ :

(a) The norm of $E$ is strongly subdifferentiable at $x$,

(b) The norm of the complexification of $E$ is strongly subdifferentiable at $x$,

(c) 1 is an isolated point of the complex triple spectrum of $x$,

(d) There exists a unique tripotent $u$ in $E$ such that $x \in E_{u}$, where

$$
E_{u}=\left\{y \in S_{E}:\{u, u, y\}=u, \quad\{u, y, u\}=u \text { and }\|y-u\|<1\right\},
$$

(e) $u\left(E^{* *}, x\right)$ belongs to $E$,

Proof. Let $\widehat{E}$ denote the complexification of $E$ (which is a complex JB*triple) and let $\tau$ the canonical conjugation on $\widehat{E}$ satisfying $\widehat{E}^{\tau}=E$. By Theorem 2.4 we already know that $(a) \Leftrightarrow(b)$ and $[3$, Theorem $2.7,(1) \Leftrightarrow(2)]$ shows the equivalence $(b) \Leftrightarrow(c)$.

To see $(b) \Rightarrow(d)$, let us suppose that the norm of the complexification, $\widehat{E}$, of $E$ is strongly subdifferentiable at $x$. By [3, Theorem $2.7,(1) \Leftrightarrow(3)]$ there exists a tripotent $u$ in $\widehat{E}$ such that $\{u, u, x\}=\{u, x, u\}=u$ and $\|x-u\|<1$. We claim that such a tripotent is unique. Indeed, suppose that $w$ is another tripotent in $\widehat{E}$ satisfying $\{w, w, x\}=\{w, x, w\}=w$ and $\|x-w\|<1$. By [14, Lemma 1.6] we have $x=u+P_{0}(u)(x)$ and $x=w+P_{0}(w)(x)$. Moreover, we also have $\left\|P_{0}(u)(x)\right\|=\|x-u\|<1$ and $\left\|P_{0}(w)(x)\right\|=\|x-w\|<1$. By Peirce rules it may be concluded that $x^{2 n+1}=u+\left(P_{0}(u)(x)\right)^{2 n+1}$ and $x^{2 n+1}=w+\left(P_{0}(w)(x)\right)^{2 n+1}$. Then we conclude that

$\|u-w\| \leq\left\|u-x^{2 n+1}\right\|+\left\|w-x^{2 n+1}\right\| \leq\left\|P_{0}(u)(x)\right\|^{2 n+1}+\left\|P_{0}(w)(x)\right\|^{2 n+1} \rightarrow 0$,

which shows that $u=w$. Therefore, there exists a unique tripotent $u \in \widehat{E}$ such that satisfying $\{u, u, x\}=\{u, x, u\}=u$ and $\|x-u\|<1$. Since $\tau$ is a conjugate-linear triple isomorphism, it follows that $\tau(u)$ is also a tripotent in $\widehat{E}$ satisfying the same conditions of $u$, thus, by the uniqueness, we have $u=\tau(u)$. This shows that $u$ is a tripotent in $E$ and $x \in E_{u}$. A similar reasoning to that just developed for the complexification shows the uniqueness of the tripotent $u$ in $E$.

$(d) \Rightarrow(e)$ Let $u$ be a tripotent in $E$ such that $x \in E_{u}$ and let $v=$ $u\left(E^{* *}, x\right)$ be the support tripotent of $x$ in $E^{* *}$. By the same reasonings 
given in the proof of Theorem 2.4 and the implication $(b) \Rightarrow(d)$ above it may be concluded that

$$
\|v-u\| \leq\left\|v-x^{2 n+1}\right\|+\left\|u-x^{2 n+1}\right\| \rightarrow 0,
$$

which shows that $v=u\left(E^{* *}, x\right)=u \in E$.

$(e) \Rightarrow(b)$ As we have seen in the comments preceding Corollary 2.3, $u\left(E^{* *}, x\right)=u\left(\widehat{E}^{* *}, x\right)$, which belongs to $E \subseteq \widehat{E}$ by hypothesis. Now [3, Theorem $2.7(4) \Rightarrow(1)$ ] ascertains that the norm of $\widehat{E}$ is strongly subdifferentiable at $x$.

We recall that a Banach space $X$ is said to be smooth at a norm-one point $u$ whenever $D(X, u)$ reduces to a singleton, and $X$ is Frechet-smooth at $u$ whenever exists the limit $\lim _{\alpha \rightarrow 0} \frac{\|u+\alpha x\|-1}{\alpha}$ for every $x \in X$ and is uniformly for $x \in B_{X}$. It is known that $X$ is Frechet-smooth at $u$ if and only if the norm of $X$ is strongly subdifferentiable at $u$ and $X$ is smooth at $u$.

The following corollary is an extension to real $\mathrm{JB}^{*}$-triples of the main result of [10] for complex $\mathrm{JB}^{*}$-triples.

Corollary 2.6. Let $E$ be a real $J B W^{*}$-triple and let $x \in S_{E}$. Then $E$ is Frechet-smooth at $x$ if and only if $E$ is smooth at $x$.

Proof. Suppose that $E$ is smooth at $x$ and let $C$ denote the real $\mathrm{JBW}^{*}$ subtriple of $E$ generated by $x$. By [5, Theorems 3.3, 3.6 and 3.7] there exist two compact hyperstonean $\Omega_{1}$ and $\Omega_{2}$ such that $C$ is isometric to

$$
C\left(\Omega_{1}, \mathbb{R}\right) \oplus^{\ell_{\infty}} C\left(\Omega_{2}, \mathbb{C}\right)_{\mathbb{R}}
$$

Since $C$ is smooth at $x=\left(x_{1}, x_{2}\right)\left(\right.$ with $x_{1} \in C\left(\Omega_{1}, \mathbb{R}\right)$ and $\left.x_{2} \in C\left(\Omega_{2}, \mathbb{C}\right)_{\mathbb{R}}\right)$ then it is easy to see that $C\left(\Omega_{1}, \mathbb{R}\right)$ is smooth at $x_{1}$ and $\left\|x_{1}\right\|=1>\left\|x_{2}\right\|$ or $C\left(\Omega_{2}, \mathbb{C}\right)_{\mathbb{R}}$ is smooth at $x_{2}$ and $\left\|x_{2}\right\|=1>\left\|x_{1}\right\|$, which implies that $C\left(\Omega_{1}, \mathbb{C}\right)$ is smooth at $x_{1}$ and $\left\|x_{1}\right\|=1$ or $C\left(\Omega_{2}, \mathbb{C}\right)$ is smooth at $x_{2}$ and $\left\|x_{2}\right\|=1$. By $\left[24\right.$, Theorem] we conclude that $C\left(\Omega_{1}, \mathbb{C}\right)$ (and hence $C\left(\Omega_{1}, \mathbb{R}\right)$ ) is Frechetsmooth at $x_{1}$ or $C\left(\Omega_{2}, \mathbb{C}\right)$ (and hence $\left.C\left(\Omega_{2}, \mathbb{C}\right)_{\mathbb{R}}\right)$ is Frechet-smooth at $x_{2}$. It can be easily seen that $C$ is Frechet-smooth at $x$. Finally the equivalence $(a) \Leftrightarrow(d)$ in Corollary 2.5 shows that $E$ is Frechet-smooth at $x$.

Remark 2.7. It should be noticed that when $X$ is a complex Banach space with a conjugation $\tau$ and $x$ is a norm-one element in $X^{\tau}$ satisfying that $X^{\tau}$ is smooth at $x$ then $X$ does not need to be smooth at $x$. For example let $X^{\tau}$ denote the real spin factor of type $I V_{n}^{n, 0}$ in the terminology of [19, Theorem 
4.1], where we consider $X$, the complexification of $X^{\tau}$, equipped with triple product and norm given by

$$
\{x y z\}:=(x \mid y) z+(z \mid y) x-(x \mid \sigma(z)) \sigma(y)
$$

and

$$
\|x\|^{2}:=(x \mid x)+\sqrt{(x \mid x)^{2}-|(x \mid \sigma(x))|^{2}},
$$

respectively, for all $x, y, z$ in $X$, where $\sigma(a+i b)=a-i b\left(a, b \in X^{\tau}\right)$ (compare [18, Theorem 4.1]). It is easy to see that any norm-one element in $X^{\tau}$ is a minimal tripotent in $X^{\tau}$ and hence $X^{\tau}$ is smooth at such a point. However, every norm-one point $x$ in $X^{\tau}$ is a tripotent that is not minimal in $X$ (the complexification of $X^{\tau}$ ), that is, there exist two orthogonal tripotents e and $f$ in $X$ such that $x=e+f$. This implies that $X$ is not smooth at $x$.

To finish with this section we will describe those real JB*-triples whose norms are strongly subdifferentiable at every point of their unit sphere. As in the complex case, we will show that such real JB*-triples are well-studied and characterized by several previous authors (compare [3, Theorem 1.12 and Remmark 2.13]).

Lemma 2.8. Let $\Omega$ be a locally compact Hausdorff space and let $\tau$ be a conjugation on $C_{0}(\Omega)$, the complex $C^{*}$-algebra of continuous complex-valued functions on $\Omega$ vanishing at infinity. Then the norm of $C_{0}(\Omega)^{\tau}$ is strongly subdifferentiable at every point of $S_{C_{0}(\Omega)^{\tau}}$ if and only if $\Omega$ is discrete.

Proof. Let us assume that the norm of $C_{0}(\Omega)^{\tau}$ is strongly subdifferentiable at every point of $S_{C_{0}(\Omega)^{\tau}}$.

By the classical Stone Theorem there exists a homomorphism $\sigma: \Omega \rightarrow \Omega$ and a continuous function $u: \Omega \rightarrow \mathbb{C}$ with $|u(t)|=1(t \in \Omega)$ such that

$$
\tau(f)(t)=u(t) \overline{f(\sigma(t))},
$$

for all $t \in \Omega, f \in C_{0}(\Omega)$. Since $\tau^{2}=I d$ we have

$$
u(t) \overline{u(\sigma(t))} f\left(\sigma^{2}(t)\right)=f(t),
$$

for all $t \in \Omega, f \in C_{0}(\Omega)$.

Let $t_{0} \in \Omega$ and let $f_{0} \in C_{0}(\Omega)$ satisfying $f_{0}\left(t_{0}\right)=f_{0}\left(\sigma^{2}\left(t_{0}\right)\right)=1$. By replacing $f_{0}$ and $t_{0}$ in (3) we deduce that

$$
u\left(t_{0}\right) \overline{u\left(\sigma\left(t_{0}\right)\right)}=1 .
$$


Since $t_{0}$ is arbitrary it follows that

$$
u(t)=u(\sigma(t)) \quad(t \in \Omega)
$$

From (3) and (4) we have $\sigma^{2}=I d_{\Omega}$.

Suppose first, that $t_{0}$ is a non-isolated point of $\Omega$. We assume first that $\sigma\left(t_{0}\right)=t_{0}$. Let $\left(U_{n}\right)$ be a sequence of compact neighbourhoods of $t_{0}$ with int $\left(U_{n}\right) \supsetneqq U_{n+1}, \sigma\left(U_{n}\right)=U_{n}$ and, by the continuity of $u$, we may also assume $\left|u(t)-u\left(t_{0}\right)\right|<\frac{1}{n}$ for all $t \in U_{n}$. Let $e_{n} \in C_{0}(\Omega)$ with $e_{n}(t)=1$ for all $t \in U_{n}$ and $e_{n}(t)=0$ for all $t \in \Omega \backslash U_{n-1}$. If $u\left(t_{0}\right) \neq-1$ we define $x=\sum_{n} \frac{1}{2^{n}} 2^{-1}\left(e_{n}+\tau\left(e_{n}\right)\right) \in C_{0}(\Omega)$. It is clear that $\tau(x)=x\left(\in C_{0}(\Omega)^{\tau}\right)$. We claim that $\lambda=\sum_{n=1}^{+\infty} \frac{1}{2^{n+1}}\left(1+u\left(t_{0}\right)\right)$ is a cluster point of the triple spectrum of $x$ in $C_{0}(\Omega)$. Indeed, for every $m \in \mathbb{N}$ we can take $t_{m} \in U_{m} \backslash U_{m+1}$, then $\lambda_{m}:=x\left(t_{m}\right)=\sum_{n=1}^{m} \frac{1}{2^{n+1}}\left(1+u\left(t_{m}\right)\right)$, is an element in the triple spectrum of $x$. We will show that $\lambda_{m}$ converges to $\lambda$. For every $\varepsilon>0$ there exists $m_{0} \in \mathbb{N}$ such that for every $m \in \mathbb{N}$ with $m \geq m_{0}$ we have

$$
\left|\lambda-\sum_{n=1}^{m} \frac{1}{2^{n+1}}\left(1+u\left(t_{0}\right)\right)\right|<\frac{\varepsilon}{2},
$$

and

$$
\begin{aligned}
\mid \sum_{n=1}^{m} \frac{1}{2^{n+1}}\left(1+u\left(t_{0}\right)\right)- & \sum_{n=1}^{m} \frac{1}{2^{n+1}}\left(1+u\left(t_{m}\right)\right)\left|\leq\left(\sum_{n=1}^{m} \frac{1}{2^{n+1}}\right)\right| u\left(t_{m}\right)-u\left(t_{0}\right) \mid \\
& <\left(\sum_{n=1}^{m} \frac{1}{2^{n+1}}\right) \frac{1}{m}<\frac{1}{m}<\frac{\varepsilon}{2} .
\end{aligned}
$$

Therefore, for every $m \geq m_{0}$ it follows that $\left|\lambda-\lambda_{m}\right|<\varepsilon$, which shows that $\lambda$ is a cluster point of the triple spectrum of $x$ in $C_{0}(\Omega)$.

When $u\left(t_{0}\right)=-1$ we define $x=\sum_{n} \frac{1}{2^{n}} 2^{-1}\left(i e_{n}+\tau\left(i e_{n}\right)\right) \in C_{0}(\Omega)^{\tau}$. Following the same method applied in the case $u\left(t_{0}\right) \neq-1$ we can conclude that $\lambda=\sum_{n=1}^{+\infty} \frac{1}{2^{n+1}} i\left(1-u\left(t_{0}\right)\right)=i \sum_{n=1}^{+\infty} \frac{1}{2^{n}}$ is a cluster point of the triple spectrum of $x$ in $C_{0}(\Omega)$.

Suppose now that $\sigma\left(t_{0}\right) \neq t_{0}$. Let $\left(U_{n}\right)$ be a sequence of compact neighbourhoods of $t_{0}$ with $\operatorname{int}\left(U_{n}\right) \supsetneqq U_{n+1}, \sigma\left(U_{n}\right) \cap U_{n}=\emptyset$ and $\left|u(t)-u\left(t_{0}\right)\right|<\frac{1}{n}$ for all $t \in U_{n}$. Let $e_{n} \in C_{0}(\Omega)$ with $e_{n}(t)=1$ for all $t \in U_{n}$ and $e_{n}(t)=0$ for all $t \in \Omega \backslash U_{n-1}$ and define $x=\sum_{n} \frac{1}{2^{n+1}}\left(e_{n}+\tau\left(e_{n}\right)\right) \in C_{0}(\Omega)$. Clearly $\tau(x)=x$. The same ideas developed in the case $\sigma\left(t_{0}\right)=t_{0}$ allow us to assure that $\lambda=\sum_{n=1}^{+\infty} \frac{1}{2^{n+1}}$ is a cluster point of the triple spectrum of $x$ in $C_{0}(\Omega)$. 
To finish the proof we claim that if a (norm-one) $\tau$-symmetric element in $C_{0}(\Omega)$ has a non discrete triple spectrum in $C_{0}(\Omega)$ then the norm of $C_{0}(\Omega)$ is not strongly subdifferentiable at an element in $S_{C_{0}(\Omega)^{\tau}}$. Indeed, let $x$ be norm-one element in $C_{0}(\Omega)$ with $\tau(x)=x$ and with non-discrete triple spectrum $S_{x} \subseteq[0,1]$. It is known that the $\mathrm{JB}^{*}$-subtriple of $C_{0}(\Omega)$ generated by $x$ (denoted by $C$ ) is $\mathrm{JB}^{*}$-triple isomorphic to the $\mathrm{C}^{*}$-algebra $C_{0}\left(S_{x}\right)$ under a triple isomorphism $\Psi$, which satisfies $\Psi(x)(t)=i d_{S_{x}}(t)=$ $t\left(t \in S_{x}\right)$. If $S_{x}$ is not discrete then there exists a non-isolated point $\alpha \in S_{x}$. The function $g(t):=\frac{t}{t+|t-\alpha|} \in C_{0}\left(S_{x}\right)$ has an odd extension to $S_{x} \cup-S_{x}$, and can be approximated uniformly by real linear combinations of odd powers of $t$. Since $\Psi^{-1}\left(i d_{S_{x}}\right)=x$ is $\tau$-symmetric and $\Psi$ is a triple isomorphism then $\Psi^{-1}(g)$ is norm-one and $\tau$-symmetric. It is easy to see that $\alpha$ is the unique $t \in S_{x}$ satisfying $g(t)=1$. Since $\alpha$ is not isolated in $S_{x}$ it follows by [10, Lemma 2.2] that the norm of $C_{0}\left(S_{x}\right)$ (and hence the norm of $C$ ) is not strongly subdifferentiable at $g$ (at $\Psi^{-1}(g)$ ), which contradicts the assumption since the strong subdifferentiability is inherited by closed subspaces.

Following [4], a real or complex $\mathrm{JB}^{*}$-triple $U$ is defined to be weakly compact if the operator $Q(a): U \rightarrow U$ defined by $Q(a)(x):=\{a, x, a\}$ is weakly compact for every $a \in U$ and to be compact if $Q(a)$ is compact for all $a \in U$. Let $E$ be a real $\mathrm{JB}^{*}$-triple with complexification $\widehat{E}$. Clearly, $E$ is weakly compact whenever $\widehat{E}$ is. On the other side, if $E$ is weakly compact, i.e. $Q(a): E \rightarrow E$ is weakly compact for every $a$ in $E$, we have $Q(a): \widehat{E} \rightarrow \widehat{E}$ is weakly compact for every $a \in E$, by [21, Theorem 10]. Let $Q(a, b): \widehat{E} \rightarrow \widehat{E}$ be the mapping given by $Q(a, b)(x):=\{a, x, b\}$. The expression $2 Q(a, b)=Q(a+b, a+b)-Q(a)-Q(a)$ implies that $Q(a, b): \widehat{E} \rightarrow$ $\widehat{E}$ is weakly compact. Since $\widehat{E}=E+i E$ and for every $x, y \in E$ the equality $Q(x+i y)=Q(x)-Q(y)+2 i Q(x, y)$ holds, we conclude that $\widehat{E}$ is weakly compact. Therefore, $E$ is weakly compact if and only if its complexification is.

Compact and weakly compact complex $\mathrm{JB}^{*}$-triples were completely described in [4, $\S 3$ and $\S 4]$. More recently, in [3, Theorem 2.12 and Remmark $2.13]$, the authors show that a complex $\mathrm{JB}^{*}$-triple is weakly compact if and only if its norm is strongly subdifferentiable at every point of its unit sphere. Our next goal is to describe those real $\mathrm{JB}^{*}$-triples whose norm is strongly subdifferentiable at every point of its unit sphere.

Theorem 2.9. Let $E$ be a real $J B^{*}$-triple. The following assertions are equivalent: 
1. The norm of the complexification, $\widehat{E}$, of $E$ is strongly subdifferentiable at every point of $S_{\widehat{E}}$.

2. The norm of $E$ is strongly subdifferentiable at every point of $S_{E}$.

3. For every $x$ in $E$, the complex triple spectrum of $x$ is discrete.

4. $\widehat{E}$ is weakly compact.

5. E is weakly compact.

Proof. Since the strong subdifferentiability at a norm-one point is inherited by subspaces, the implication $(1.) \Rightarrow(2$.) is clear.

(2.) $\Rightarrow$ (3.) Let $\widehat{E}$ denote the complexification of $E$ and let $\tau$ be the canonical conjugation on $\widehat{E}$ satisfying $\widehat{E}^{\tau}=E$. By Theorem 2.4 we conclude that the norm of $\widehat{E}$ is strongly subdifferentiable at every point $x$ in $S_{E}$ $(\|x\|=1$ and $\tau(x)=x)$. Let $x$ be a norm-one element in $E$ and let $\widehat{E}(x)$ denote the complex $\mathrm{JB}^{*}$-subtriple of $\widehat{E}$ generated by $x$. Since $\tau(x)=x$ we deduce that $\left.\tau\right|_{\widehat{E}(x)}$ is a conjugation on $\widehat{E}(x)$. Since the strong subdifferentiability is inherited by subspaces we conclude that the norm of $\widehat{E}(x)$ is strongly subdifferentiable at every point $x \in S_{\widehat{E}(x)^{\tau}}$. It is known that $\widehat{E}(x)$ is $\mathrm{JB}^{*}$-triple isomorphic to the $\mathrm{C}^{*}$-algebra $C_{0}\left(S_{x}\right)$, therefore Lemma 2.8 implies that $S_{x}$ is discrete.

$(3.) \Rightarrow(4$.) Since the implication $(3$.) $\Rightarrow(2$.) follows by Corollary 2.5 , we deduce that $(3.) \Leftrightarrow(2$.). We therefore assume that the norm of $E$ is strongly subdifferentiable at every point of $S_{E}$.

Let $z$ be in $E$ and let $E(z)$ denote the real JB*-subtriple of $E$ generated by $z$. By Zorn's lemma there is an abelian subtriple $C$ containing $E(z)$ which is maximal with respect to inclusion. Let $\widehat{C}$ denote the complexification of $C$. Since $C$ is abelian then $\widehat{C}$ is an abelian $\mathrm{JB}^{*}$-triple. It is well known that $\widehat{C}$ is triple isomorphic (and hence isometric) to $C_{0}(\Omega)$ for some locally compact Hausdorff space $\Omega$. Since the strong subdifferentiability is inherited by subspaces it follows that the norm of $\widehat{C}$ is strong subdifferentiable at every point in $S_{C}$, by Theorem 2.4. Now Lemma 2.8 implies that $\Omega$ is discrete and hence there exists a family, $\left\{e_{\alpha}\right\}$, of mutually orthogonal minimal tripotents in $C$ such that every element in $C$ can be approximated in norm by linear combinations of $\left\{e_{\alpha}\right\}$. We claim that every $e_{\alpha}$ is also a minimal tripotent in $E$. Suppose on the contrary that there exists $0 \neq x \in E^{1}\left(e_{\alpha_{0}}\right) \backslash \mathbb{R} e_{\alpha_{0}}$, for some $\alpha_{0}$. Let $C^{\prime}$ denote the real $\mathrm{JB}^{*}$-subtriple generated by $C$ and $x$. Since for every $\alpha \neq \beta$ we have $e_{\alpha} \perp e_{\beta}$ we conclude that $C^{\prime}$ is an abelian real $\mathrm{JB}^{*}$-triple containing $C$ which contradicts the maximality of $C$. 
Therefore, every element in $E$ can be approximated in norm by linear combinations of minimal tripotents in $E$. Since $\widehat{E}=E+i E$ we conclude, by [22, Lemma 3.2] (see also [2, Corollary 3.5]), that every element in $\widehat{E}$ can be approximated in norm by linear combinations of minimal tripotents in $\widehat{E}$. By $[4$, Theorem $3.4,(i) \Leftrightarrow(v i)]$ it follows that $\widehat{E}$ is weakly compact.

As we have seen in the comments preceding this Theorem, $(4) \Leftrightarrow(5)$. Finally $(4) \Leftrightarrow(1)$, by [3, Theorem 2.12 and Remmark 2.13].

\section{Applications}

The aim of this section is to obtain an alternative proof of Kaup's BanachStone Theorem for JB*-triples by applying the characterization of the points of strong subdifferentiability for the norm of a JB*-triple. This provides a complete proof to the statement settled by W. Werner in [23, Remarks 3.] and extend the method developed in the already quoted paper to the more general setting of $\mathrm{JB}^{*}$-triples.

Having in mind that the bidual of every real or complex $\mathrm{JB}^{*}$-triple $E$ is a real or complex $\mathrm{JBW}^{*}$-triple, and since every tripotent in $E$ is also a tripotent in its bidual, the proof of the following Lemma could be derived from [11, Lemma 2.1] and [11, Theorem 3.7] in the complex and real case, respectively.

Lemma 3.1. Let $E$ be a real or complex $J B^{*}$-triple, and let e, $u$ be tripotents in $E$. If $D(E, e)$ coincides with $D(E, u)$, then $e=u$.

The following lemma generalizes [23, Lemma 3] to the setting of real and complex JB*-triples.

Lemma 3.2. Let $E$ be a real or complex $J B^{*}$-triple, let e be a tripotent in $E$ and $a \in S_{E}$. The following statements hold:

(a) $a \in E_{e}$ if, and only if, $D(E, a)=D(E, e)$.

(b) If $x \in E_{e}$ satisfies $\|x-b\|<1$ for all $b \in E_{e}$ then $x=e$.

Proof. Suppose first that $E$ is a complex JB*-triple.

(a) $(\Rightarrow)$ Suppose $a \in E_{e}$. In particular we have $\{e, a, e\}=\{e, e, a\}=e$, which implies $P_{2}(e)(a)=e$. Since $\|a\|=1,[14$, Lemma 1.6] assures that $P_{1}(e)(a)=0$, and hence $a=e+z_{0}$, where $z_{0} \in E_{0}(e)$. Let $f \in D(E, e)$. By [14, Proposition 1] we have $f=f P_{2}(e)$ and $f(a)=f P_{2}(e)(a)=f(e)=1$. This implies $f \in D(E, a)$, and hence $D(E, e) \subseteq D(E, a)$. 
To see the other inclusion, let $C$ be the $\mathrm{JB}^{*}$-subtriple generated by $e$ and $a=e+z_{0}\left(z_{0} \in E_{0}(e),\left\|z_{0}\right\|<1\right)$. Since, by the Peirce arithmetic, $e$ and $z_{0}$ are orthogonal and $e$ is a tripotent, it follows that $C$ coincides with $\mathbb{C} e \oplus^{\infty} D$, where $D$ is the $\mathrm{JB}^{*}$-subtriple generated by $z_{0}$ which is triple isomorphic (and hence isometric) to $C_{0}\left(S_{z_{0}}\right)$. Therefore, $C$ is triple isomorphic (and hence isometric) to an abelian $\mathrm{C}^{*}$-algebra.

According with the notation of [23, Theorem 4] we can see that

$$
a \in C_{e}=F_{e, 0}(C):=\left\{y \in S_{C}: y e^{*}=e e^{*} \text { and }\|y-e\|<1\right\} .
$$

Therefore, by [23, Lemma 3], we have $D(C, e)=D(C, a)$. Finally, let $f$ in $D(E, a)$. It is clear that $\left.f\right|_{C}$ lies in $D(C, a)=D(C, e)$ and hence $f(e)=1$. This assures that $D(E, a) \subseteq D(E, e)$.

$(\Leftarrow)$ Suppose now that $D(E, a)=D(E, e)$. By [3, Theorem 2.7] we conclude that the norm of $E$ is strongly subdifferentiable at every tripotent element of $E$. Since the strong subdifferentiability of the norm of $E$ at a norm-one element $x$ depends only on the set $D(E, x)$ (compare [13, Theorem 1.2 and Proposition 3.1]) it follows that the norm of $E$ is also strongly subdifferentiable at $a$.

From [3, Theorem 2.7] we conclude that there is a tripotent $u$ in $E$ such that $a$ lies in $E_{u}$. By the first part of the proof we have $D(E, u)=D(E, a)=$ $D(E, e)$. By Lemma 3.1 we get $u=e$, which gives $a \in E_{e}$.

(b) Let $C$ be the $\mathrm{JB}^{*}$-subtriple generated by $e$ and $x=e+z_{0}\left(z_{0} \in E_{0}(e)\right.$, $\left.\left\|z_{0}\right\|<1\right)$. As we have seen in the first part of the proof, $C$ is an abelian $\mathrm{C}^{*}$-algebra. By hypothesis we have $x \in C_{e}=F_{e, 0}(C)$ and $\|x-b\|<1$ for every $b \in C_{e}=F_{e, 0}(C)$. By $[23$, Lemma $3(i i)]$ we get $x=e$.

Suppose now that $E$ is a real $\mathrm{JB}^{*}$-triple. Having in mind that [14, Lemma 1.6, and Proposititon 1] remain true for real $\mathrm{JB}^{*}$-triples, the proof of $(a)$ is a repetition of the one given in the complex case but replacing [3, Theorem 2.7] by Corollary 2.5. To see $(b)$ let $\widehat{E}$ denote the complexification of $E$ (which is a complex $\mathrm{JB}^{*}$-triple) and let $\tau$ denote the canonical conjugation on $\widehat{E}$ such that $\widehat{E}^{\tau}=E$. Suppose $x \in E_{e}$ satisfies $\|x-b\|<1$ for all $b \in E_{e}$.

As we have seen several times $x=e+z_{0}$, where $z_{0} \in E_{0}(e)$, and clearly $x \in \widehat{E}_{e}$. Let $c \in \widehat{E}_{e}$ then $c=b_{1}+i b_{i}$ for some $b_{1}, b_{2} \in E$. The equalities $\{e, c, e\}=\{e, e, c\}=e=\tau(e)$ give us

$$
\left\{e, b_{1}, e\right\}=\left\{e, e, b_{1}\right\}=e, \quad\left\{e, e, b_{2}\right\}=\left\{e, b_{2}, e\right\}=0 .
$$

Therefore $b_{1} \in E^{1}(e)=\widehat{E}^{1}(e) \subset \widehat{E}_{2}(e)$ and $b_{2} \in E_{0}(e) \subset \widehat{E}_{0}(e)$. By [14, Lemma 1.3] it follows

$$
1=\|c\|=\left\|b_{1}+i b_{2}\right\|=\max \left\{\left\|b_{1}\right\|,\left\|b_{2}\right\|\right\},
$$




$$
1>\|e-c\|=\left\|e-b_{1}-i b_{2}\right\|=\max \left\{\left\|e-b_{1}\right\|,\left\|b_{2}\right\|\right\},
$$

and then $\left\|e-b_{1}\right\|,\left\|b_{2}\right\|<1$. It is easy to see that

$$
1=\|e\|=\left\|\left\{e, b_{1}, e\right\}\right\| \leq\left\|b_{1}\right\|,
$$

which shows $b_{1} \in E_{e}$. By hypothesis $\left\|x-b_{1}\right\|<1$. Finally

$$
\|x-c\|=\left\|x-b_{1}-i b_{2}\right\|=\max \left\{\left\|x-b_{1}\right\|,\left\|b_{2}\right\|\right\}<1,
$$

for every $c \in \widehat{E}_{e}$. Now, the proof in the complex case assures that $x=e$.

Corollary 3.3. Let $\Phi: E \rightarrow F$ be a surjective isometry between two real or complex JB*-triples. Then $\Phi$ preserves tripotents.

Proof. Let $e$ be a tripotent in E. By Corollary 2.5, it follows that the norm of $E$ is strongly subdifferentiable at $e$. Since the strong subdifferentiability is preserved by surjective isometries, we can conclude that the norm of $F$ is strongly subdifferentiable at $\Phi(e)$. By Corollary $2.5(d)$, there is a (unique) tripotent $u$ in $F$ such that $\Phi(e) \in F_{u}$.

Let $x \in E_{e}$. By Lemma $3.2(a)$ we deduce that

$$
D(F, \Phi(x))=\left(\Phi^{*}\right)^{-1} D(E, x)=\left(\Phi^{*}\right)^{-1} D(E, e)=D(F, \Phi(e))=D(F, u) .
$$

Again Lemma $3.2(a)$, implies $x \in F_{u}$. Therefore $\Phi\left(E_{e}\right) \subseteq F_{u}$. Similar arguments show the reciprocal inclusion and the equality $\Phi\left(E_{e}\right)=F_{u}$.

Given $y \in F_{u}=\Phi\left(E_{e}\right)$, there is $x \in E_{e}$ with $\Phi(x)=y$, thus

$$
\|y-\Phi(e)\|=\|\Phi(x)-\Phi(e)\|=\|\Phi(x-e)\|=\|x-e\|<1 .
$$

Now, Lemma $3.2(b)$, gives us $\Phi(e)=u$. This shows that $\Phi$ preserves tripotents.

It is well known that the fact that any surjective isometry between complex $\mathrm{JB}^{*}$-triples preserves tripotents can be applied to give an alternative proof to Kaup's Banach-Stone Theorem (see for instance [7] or [12, Proof of Theorem 2.2]).

Corollary 3.4. Let $\Phi: E \rightarrow F$ be a surjective isometry between two complex $J B^{*}$-triples. Then $\Phi$ is a triple isomorphism.

Acknowledgments: The authors would like to thank A. Rodríguez Palacios for fruitful comments and discussions during the preparation of this paper and to the referee for his or her interesting suggestions. 


\section{References}

[1] T. J. Barton and R. M. Timoney, Weak*-continuity of Jordan triple products and applications. Math. Scand. 59 (1986), 177-191.

[2] J. Becerra-Guerrero, G. López, A. M. Peralta and A. RodríguezPalacios, Relatively weakly open sets in closed unit balls of Banach spaces, and real JB*-triples of finite rank, preprint 2003.

[3] J. Becerra-Guerrero and A. Rodríguez-Palacios, Strong subdifferentiability of the norm on JB*-triples, Quart. J. Math. Oxford. 54, 381-390 (2003).

[4] L. J. Bunce and C.-H. Chu, Compact operations, multipliers and Radon-Nikodým property in JB*-triples, Pacific J. Math. 153, no. 2, 249-265 (1992).

[5] L. J. Bunce, C.-H. Chu, Real contractive projections on commutative $C^{*}$-algebras, Math. Z. 226, no. 1, 85-101 (1997).

[6] M. D. Contreras, R. Payá, and W. Werner, $C^{*}$-algebras that are $I$-rings, J. Math. Anal. Appl. 198, no. 1, 227-236 (1996).

[7] T. Dang, Y. Friedman and B. Russo, Affine geometric proofs of the Banach Stone theorems of Kadison and Kaup. Proceedings of the Seventh Great Plains Operator Theory Seminar (Lawrence, KS, 1987), Rocky Mountain J. Math. 20, no. 2, 409-428 (1990).

[8] S. Dineen, The second dual of a JB*-triple system, In: Complex analysis, functional analysis and approximation theory (ed. by J. Múgica), 67-69, (North-Holland Math. Stud. 125), North-Holland, AmsterdamNew York, 1986.

[9] C. M. Edwards and G. F. Rüttimann, On the facial structure of the unit balls in a JBW*-triple and its predual, J. London Math. Soc. (2) 38, no. 2, 317-332 (1988).

[10] C. M. Edwards and G. F. Rüttimann, Smoothness properties of the unit ball in a JB*-triple, Bull. London Math. Soc. 28, 156-160 (1996).

[11] C. M. Edwards and G. F. Rüttimann, The facial and inner ideal structure of a real JBW*-triple, Math. Nach. 222, 159-184 (2001). 
[12] F. J. Fernandez-Polo, J. Martínez Moreno and A. M. Peralta, Geometric characterization of tripotents in real and complex $\mathrm{JB}^{*}$-triples, preprint 2003.

[13] C. Franchetti and R. Payá, Banach spaces with strongly subdifferentiable norm, Bolletino U. M. I. 7-B, 45-70 (1993).

[14] Y. Friedman and B. Russo, Structure of the predual of a JBW*-triple, J. Reine u. Angew. Math. 356, 67-89 (1985).

[15] J. R. Giles, D. A. Gregory and B. Sims, Geometrical implications of upper semi-continuity of the duality mapping on a Banach space, Pacific J. Math. 79, no. 1, 99-109 (1978).

[16] J. M. Isidro, W. Kaup, and A. Rodríguez, On real forms of JB*-triples, Manuscripta Math. 86, 311-335 (1995).

[17] W. Kaup, Algebraic Characterization of symmetric complex Banach manifolds, Math. Ann. 228, 39-64 (1977).

[18] W. Kaup, A Riemann Mapping Theorem for bounded symmentric domains in complex Banach spaces, Math. Z. 183, 503-529 (1983).

[19] W. Kaup, On real Cartan factors, Manuscripta Math. 92, 191-222 (1997).

[20] J. Martínez and A. M. Peralta, Separate weak*-continuity of the triple product in dual real JB*-triples, Math. Z. 234, 635-646 (2000).

[21] A. M. Peralta and A. Rodríguez Palacios, Grothendieck's inequalities for real and complex JBW*-triples, Proceedings of the London Math. Soc. (3) 83, 605-625 (2001).

[22] A. M. Peralta and L.L. Stacho, Atomic decomposition of real JBW*triples, Quart. J. Math. Oxford 52, 79-87 (2001)

[23] W. Werner, Subdifferentiability and the Noncommutative BanachStone Theorem, In Function spaces (Edwardsville, IL, 1994), 377-386, Lecture Notes in Pure and Appl. Math., 172, Dekker, New York, 1995.

[24] K. F. Taylor and W. Werner, Differentiability of the norm in von Neumann algebras, Proc. Amer. Math. Soc. 119, no. 2, 475-480 (1993). 